\title{
Flavor structure of the baryon-baryon interaction from lattice QCD
}

\section{Takashi Inoue*}

Nihon University, College of Bioresource Sciences, Fujisawa 252-0880, Japan

E-mail: inoue.takashi@nihon-u.ac.jp

\section{for HAL QCD Collaboration}

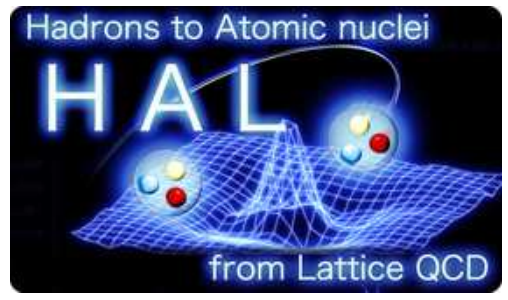

\begin{abstract}
We investigate baryon-baryon $(B B)$ interactions in the 3-flavor full QCD simulations with degenerate quark masses for all flavors. The $B B$ potentials in the orbital $S$-wave are extracted from the Nambu-Bethe-Salpeter wave functions measured on the lattice. We observe strong flavor-spin dependences of the $B B$ potentials at short distances. In particular, a strong repulsive core exists in the flavor-octet and spin-singlet channel (the $\mathbf{8}_{s}$ representation), while an attractive core appears in the flavor singlet channel (the $\mathbf{1}$ representation). We discuss the relation of such flavor-spin dependence with the Pauli exclusion principle at the quark level. The possible existence of an $H$-dibaryon resonance above the $\Lambda \Lambda$ threshold is also discussed.
\end{abstract}

The XXVIII International Symposium on Lattice Field Theory, Lattice2010

June 14-19, 2010

Villasimius, Italy

\footnotetext{
* Speaker.
} 


\section{Introduction}

The generalized nuclear force, which includes not only the nucleon-nucleon $(N N)$ interaction but also hyperon-nucleon $(Y N)$ and hyperon-hyperon $(Y Y)$ interactions, has been one of the central topics in hadron and nuclear physics. Experimental studies on the ordinary and hyper nuclei as well as the observational studies of the neutron stars and supernova explosions are deeply related to the physics of the generalized nuclear force [1].

Concerning the $N N$ interaction, significant number of scattering data have been accumulated and are parametrized in terms of the phase shifts or of the phenomenological potentials [2]. On the other hand, the properties of the $Y N$ and $Y Y$ interactions are hardly known even today due to the lack of high precision $Y N$ and $Y Y$ scattering data. The approximate flavor $S U(3)$ symmetry does not necessarily create a bridge between the nuclear force and the hyperon forces because there are six independent flavor-channels for the scatterings between octet-baryons. Although there have been previous theoretical attempts to fill the gap on the basis of the phenomenological quark models [3] and of the one-boson-exchange models [4], it is much more desirable to study the generalized nuclear force from the first principle QCD.

Recently a method to extract the interaction potential from lattice QCD simulations has been proposed and applied to the $N N$ system [5], and $\Xi N$ and $\Lambda N$ systems [6] as well. This paper is our exploratory attempt to unravel the flavor-spin structure of the $B B$ interaction on the basis of the lattice QCD simulations[7]. To capture the essential structure, we take the finite but degenerated u, d, s-quark masses, so that the system is in the flavor $S U(3)$ limit. In the limit a convenient base set exist to look into the structure. Moreover, $S U(3)$ limit result is easy to compare with effective models and useful to pin down physical origin of the $B B$ interaction.

In the next section, we introduce the flavor $S U(3)$ limit and the formalism we employ. In section 3, we explain a setup of our numerical simulations. In section 4 , we show the resulting $B B$ potentials and discuss their implications. The last section is devoted to summary and outlook.

\section{Baryon-baryon potentials in the flavor $S U(3)$ limit}

In the flavor $S U(3)$ symmetric limit, all baryons and baryonic systems are strictly classified into multiplets which serve irreducible representation of the flavor $S U(3)$ group. For example, two-octet-baryon systems are classified as

$$
\mathbf{8} \otimes \mathbf{8}=\underbrace{\mathbf{2 7} \oplus \mathbf{8}_{s} \oplus \mathbf{1}}_{\text {symmetric }} \oplus \underbrace{\mathbf{1 0}^{*} \oplus \mathbf{1 0} \oplus \mathbf{8}_{a}}_{\text {antisymmetric }}
$$

where "symmetric" and "antisymmetric" stand for the symmetry under the flavor exchange of two baryons. The Pauli principle between two baryons imposes, for the system with even (odd) orbital angular momentum, 27, $\mathbf{8}_{s}$ and $\mathbf{1}$ to be spin singlet (triplet), while $\mathbf{1 0}^{*}, \mathbf{1 0}$ and $\mathbf{8}_{a}$ to be spin triplet (singlet). These six states provide good basis to describe $B B$ interaction. In particular, for $S$-wave, all off-diagonal interactions vanish and six diagonal one remain. The corresponding potentials are

$$
\begin{aligned}
& { }^{1} S_{0}: V^{(\mathbf{2 7})}(r), V^{\left(\mathbf{8}_{s}\right)}(r), V^{(\mathbf{1})}(r), \\
& { }^{3} S_{1}: V^{\left(\mathbf{1 0}^{*}\right)}(r), V^{(\mathbf{1 0})}(r), V^{\left(\mathbf{8}_{a}\right)}(r) .
\end{aligned}
$$


Table 1: Summary of lattice parameters and hadron masses. For details about the action and the lattice spacing $a$, visit the official website of CP-PACS and JLQCD Collaborations[10].

\begin{tabular}{c|c|c|c|c|c|c|c}
\hline \hline lattice & $\beta$ & $a[\mathrm{fm}]$ & $\mathrm{L}[\mathrm{fm}]$ & $\kappa_{u d s}$ & $m_{\mathrm{ps}}[\mathrm{MeV}]$ & $m_{B}[\mathrm{MeV}]$ & $N_{\mathrm{cfg}}$ \\
\hline \multirow{2}{*}{$16^{3} \times 32$} & \multirow{2}{*}{1.83} & \multirow{2}{*}{$0.121(2)$} & \multirow{2}{*}{$1.93(3)$} & 0.13710 & $1014(1)$ & $2026(3)$ & 700 \\
& & & & 0.13760 & $835(1)$ & $1752(3)$ & 800 \\
\hline \hline
\end{tabular}

These six potentials contain the essential structure of $S$-wave $B B$ interaction. In fact, any potential among octet-baryons, including both the diagonal one $\left(B_{1} B_{2} \rightarrow B_{1} B_{2}\right)$ and the off-diagonal one $\left(B_{1} B_{2} \rightarrow B_{3} B_{4}\right)$, is obtained by suitable combinations of these six potentials.

According to Refs. [5], non-local but energy-independent potential can be defined and extracted in lattice QCD, from the Nambu-Bethe-Salpeter (NBS) wave function $\phi(\vec{r})$ and the energy $E$, through the Schrödinger type equation. As the leading order of the derivative expansion of the non-local potential, a local potential is obtained by

$$
V^{(\alpha)}(\vec{r})=\frac{1}{2 \mu} \frac{\nabla^{2} \phi^{(\alpha)}(\vec{r})}{\phi^{(\alpha)}(\vec{r})}+E^{(\alpha)}
$$

where $\mu$ is the reduced mass of the system. It is shown that the leading local potential well dominate the full potential at low energy region [8]. We use eq. (2.3) and obtain the six potentials in eq. (2.2). For ${ }^{3} S_{1}$ states, we do not decompose central and tensor potential in this study, and hence the resulting potential is the one so called "effective central" potential.

In the lattice QCD simulations, the NBS wave function $\phi^{(\alpha)}(\vec{r})$ for the smallest energy is extracted from the four-point function as

$$
G_{4}^{(\alpha)}\left(t-t_{0}, \vec{r}\right)=\left\langle 0\left|(B B)^{(\alpha)}(t, \vec{r}) \overline{(B B)}(\alpha)\left(t_{0}\right)\right| 0\right\rangle \propto \phi^{(\alpha)}(\vec{r}) e^{-W\left(t-t_{0}\right)}
$$

for $t-t_{0} \gg 1$, where $\overline{(B B)}^{(\alpha)}\left(t_{0}\right)$ is a wall source operator at time $t_{0}$ to create two-baryon states in $\alpha$-plet, while $(B B)^{(\alpha)}(t, \vec{r})$ is the sink operator at time $t$ to annihilate two-baryon states. These two-baryon operators can be constructed with product of the baryon composite field operator and the $S U$ (3) Clebsch-Gordan (CG) coefficients.

\section{Numerical simulations}

We utilize two gauge configuration sets provided by the Japan Lattice Data Grid (JLDG) and International Lattice Data Grid (ILDG) [9], which are generated by CP-PACS and JLQCD Collaborations [10], with the renormalization-group-improved Iwasaki gauge action and the nonperturbatively $O(a)$-improved Wilson quark action. The lattice parameters and hadron masses are summarized in Table 1 . Those hopping parameters $\kappa_{u d s}$ reside around the physical strange quark mass region in $2+1$ flavor QCD at the same $\beta[10]$.

We calculate quark propagators for the spatial wall source at $t_{0}$ with the Dirichlet boundary condition in the temporal direction at $t=t_{0}+16$. In order to enhance the signal, we use all 32 time slices on each configuration as $t_{0}$, and take the average over forward and backward propagations in time. We estimate the statistical errors by the jackknife method. All these numerical computations have been done at the KEK supercomputer system, Blue Gene/L and SR11000. 

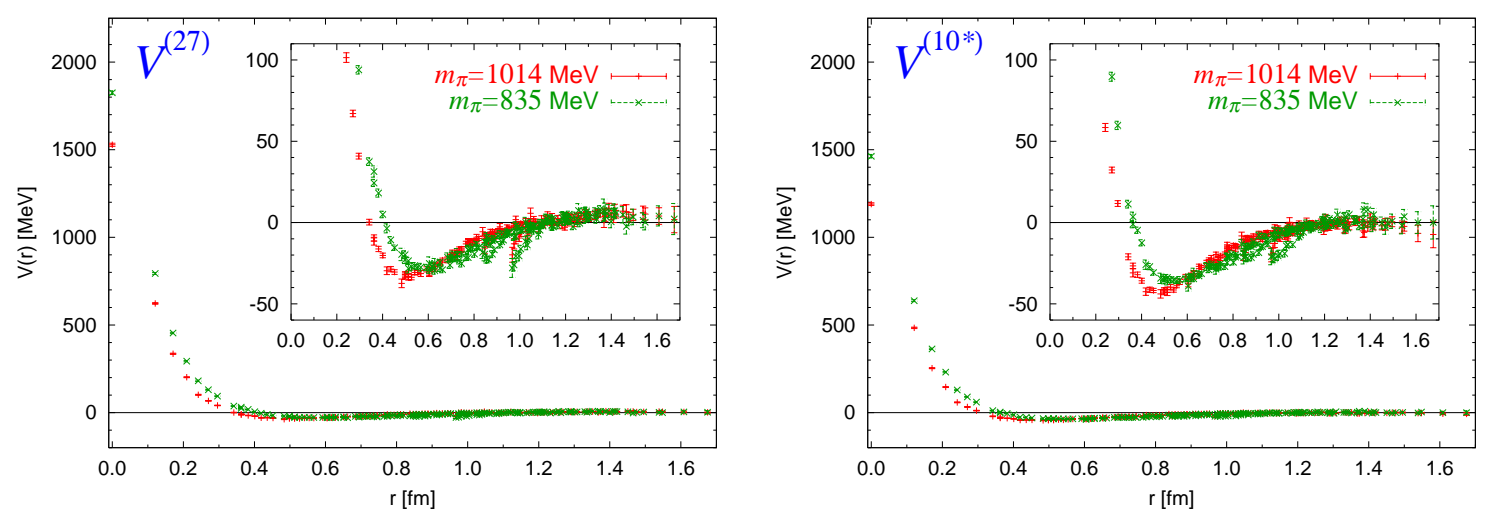

Figure 1: Lattice QCD extracted potentials $V^{(27)}$ in the left and $V^{\left(\mathbf{1 0}^{*}\right)}$ in the right.

\section{Results and their implications}

\section{1 $B B$ potentials in flavor basis}

Figure 1 to 3 show the obtained six $B B$ potentials. Red bars (green crosses) correspond to the pion mass of $1014 \mathrm{MeV}$ ( $835 \mathrm{MeV})$. Since the effective mass of the four-point function in each channel shows a plateau at $t-t_{0} \geq 10$, we use data at $t-t_{0}=10$ exclusively to extract both NBS wave functions and $B B$ potentials throughout this paper. We have estimated the energies contribution (the second term on the right-hand side of Eq. (2.3)) under the condition that Eq. (2.3) approaches zero at large $r$ (the method called "from $V$ " in Ref. [5]). We find $E^{(27)} \simeq-5 \mathrm{MeV}$, $E^{\left(\mathbf{(}_{s}\right)} \simeq 25 \mathrm{MeV}, E^{(\mathbf{1})} \simeq-30 \mathrm{MeV}, E^{\left(\mathbf{1 0}^{*}\right)} \simeq-10 \mathrm{MeV}, E^{(\mathbf{1 0})} \simeq 0 \mathrm{MeV}$ and $E^{\left(\mathbf{8}_{a}\right)} \simeq-15 \mathrm{MeV}$. Since the energy contribution is small compared to the Laplacian term (the first term on the righthand side of Eq. (2.3)) at a short distance, we have not attempted to extract a precise value of $E^{(\alpha)}$ in this study.

Figure 1 shows potentials $V^{(\mathbf{2 7})}$ and $V^{\left(\mathbf{1 0}^{*}\right)}$. Because a symmetric $N N$ belongs to $\mathbf{2 7}$ and antisymmetric $N N$ belongs to $\mathbf{1 0}^{*}$ in the $S U(3)$ limit, $V^{(\mathbf{2 7})}$ and $V^{\left(\mathbf{1 0}^{*}\right)}$ can be regarded as the $S U(3)$ limit of the nuclear force potential in ${ }^{1} S_{0}$ and ${ }^{3} S_{1}$, respectively. Both potential have a repulsive core at a short distance and an attractive pocket at around $0.6 \mathrm{fm}$. These qualitative features agree with our previous results found in the $N N$ system in quenched approximation with lighter quark mass[5]. This gives good consistency check. Furthermore, these feature qualitatively agree with well known property of the phenomenological $N N$ potentials constructed form data. This shows efficiency of the present method.

Figure 2 shows potentials $V^{(\mathbf{1 0})}$ and $V^{\left(\mathbf{8}_{a}\right)}$. Since anti-symmetric $N \Sigma(I=3 / 2)$ belongs to $\mathbf{1 0}$ in the $S U$ (3) limit, one can regard $V^{(\mathbf{1 0})}$ as the $S U(3)$ limit of ${ }^{3} S_{1} N \Sigma(I=3 / 2)$ potential, for example. Similarly, $V^{\left(\mathbf{8}_{a}\right)}$ is the $S U(3)$ limit of ${ }^{3} S_{1} N \Xi(I=0)$ potential. As you see, $V^{(\mathbf{1 0})}$ has a stronger repulsive core and a much shallower attractive pocket than $V^{\left(\mathbf{2 7}, \mathbf{1 0}^{*}\right)}$. On the other hand, $V^{\left(\mathbf{8}_{a}\right)}$ has

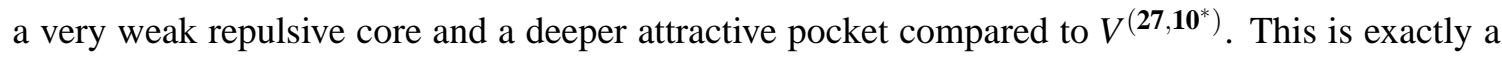
part of the essential structure of $B B$ interaction we looked for. It is interesting to point out that the above features were foreseen in a simple quark model, where effect of quark Pauli blocking and one-gluon-exchange interaction are evaluated systematically using the $S U(6)$ quark wave function of baryon[3]. We come back this point later. 

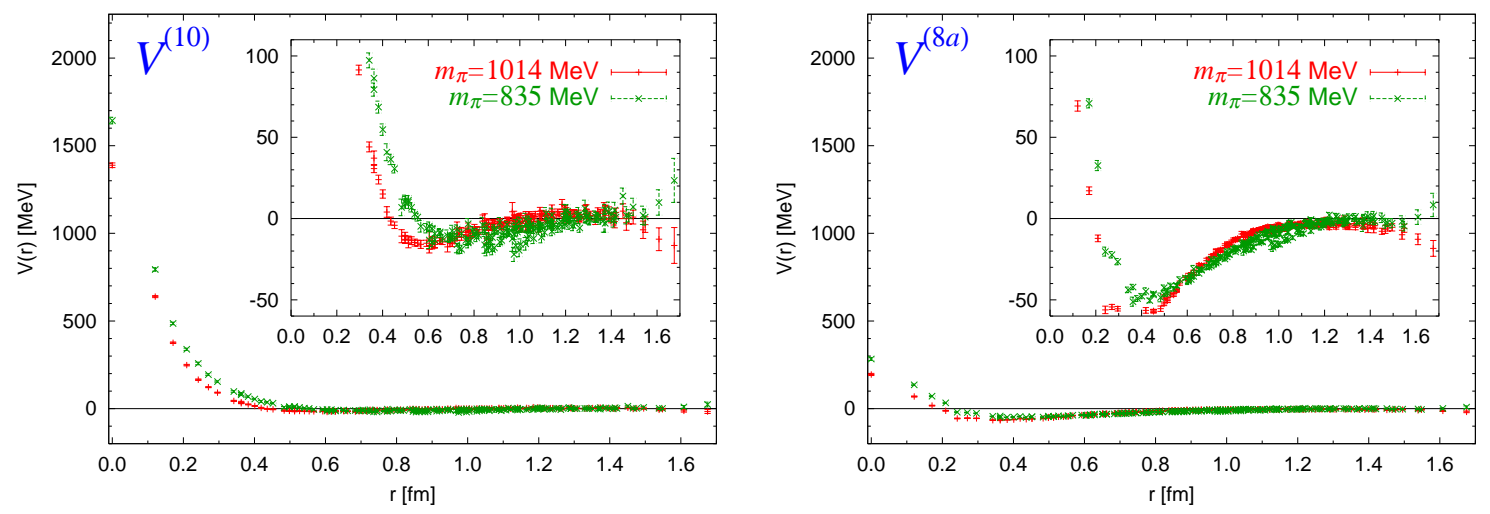

Figure 2: Lattice QCD extracted potentials $V^{(\mathbf{1 0})}$ in the left and $V^{\left(\mathbf{8}_{a}\right)}$ in the right.
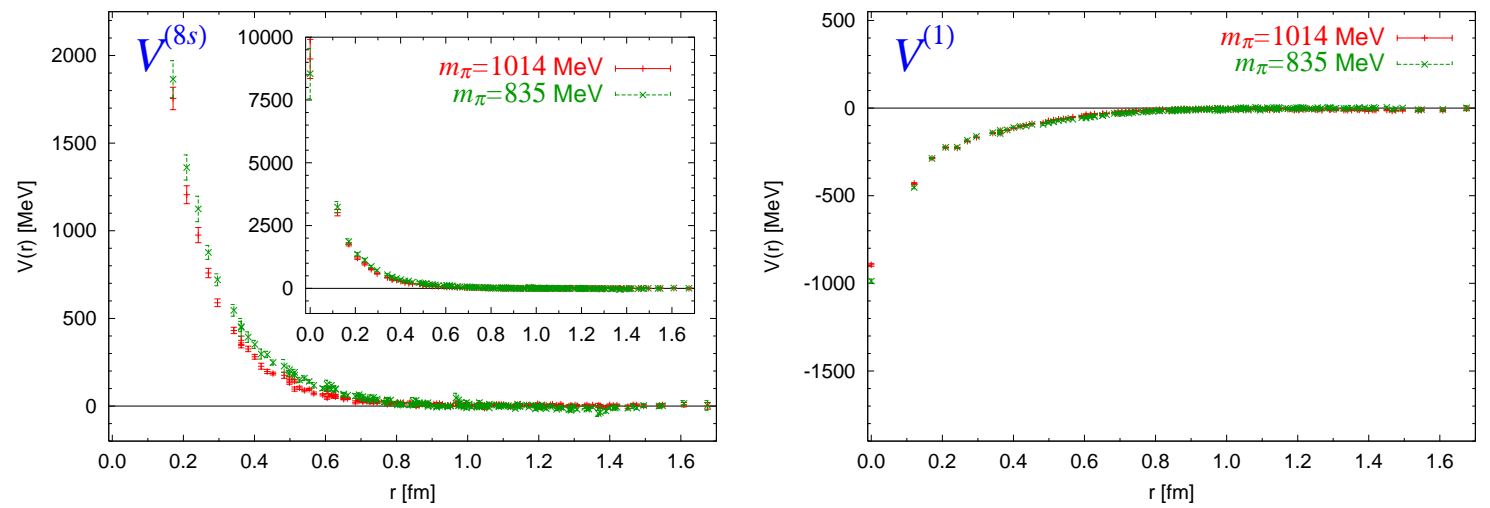

Figure 3: Lattice QCD extracted potentials $V^{\left(\mathbf{8}_{s}\right)}$ in the left and $V^{(\mathbf{1})}$ in the right.

Figure 3 shows potentials $V^{(\mathbf{8 s})}$ and $V^{(\mathbf{1})}$. These potentials cannot be interpreted as an $S U(3)$ limit of some potential between two baryons, but are always superpositions of them. First, we see that $V^{\left(\mathbf{8}_{s}\right)}$ has a very strong repulsive core. It is strongest among all the channels. On the other hand, $V^{(\mathbf{1})}$ shows no repulsion at a short distance but attraction instead. It is in contrast to other cases. Now, the essential flavor-spin structure of the $S$-wave $B B$ interaction is revealed completely.

As the case of $V^{(\mathbf{1 0})}$ and $V^{\left(\mathbf{8}_{a}\right)}$, the above features of $V^{\left(\mathbf{8}_{s}\right)}$ and $V^{(\mathbf{1})}$ were also foreseen in simple quark models[3, 11]. In particular, $V^{\left(\mathbf{8}_{s}\right)}$ in quark models becomes strongly repulsive at a short distance since the six quarks cannot occupy the same orbital state due to quark Pauli blocking. On the other hand, in the $\mathbf{1}$ channel six quarks do not suffer from the quark Pauli blocking at all, and the potential can become attractive due to short-range gluon exchange. In total, simple quark models predicted the essential feature of $B B$ interaction correctly. This fact suggests that the quark Pauli blocking plays an essential role in the repulsion in $B B$ system.

\section{2 $B B$ potentials in baryon basis}

With explicit flavor $S U(3)$ breaking, the baryon basis such as $N N$ and $\Lambda N$ are more suitable to describe $B B$ interactions than the flavor basis. Generally, interaction potential takes matrix form in coupled channels in the baryon basis. In the $S U(3)$ limit, the baryon-base potentials can be 

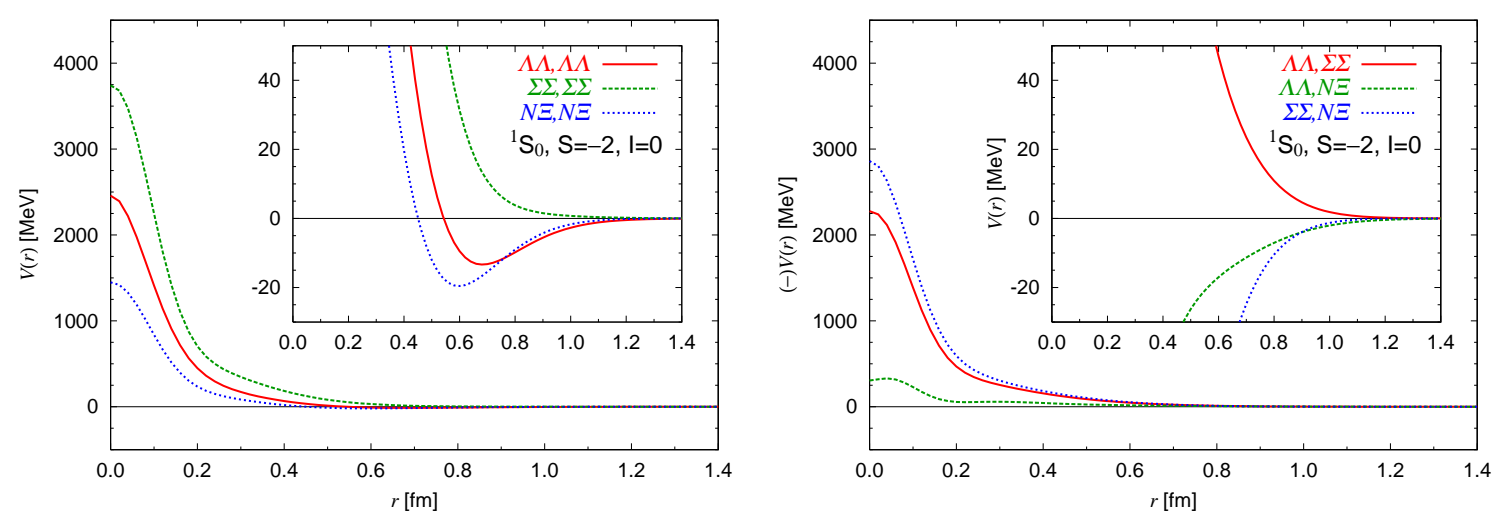

Figure 4: $B B$ potentials in baryon basis for $S=-2, I=0,{ }^{1} S_{0}$ sector. Three diagonal (off-diagonal) potentials are shown in the left (right) panel. Phases of off-diagonal ones in the right panel are arranged in a zoom-out plot. Their true signs are shown in the insert.

obtained by a unitary rotation of flavor-base potentials as $V_{i j}(r)=\sum_{\alpha} U_{i \alpha} V^{(\alpha)}(r) U_{\alpha j}^{\dagger}$ where $U$ is a unitary matrix made of CG coefficients, which rotates the flavor basis to the baryon basis.

In Figure 4, we show the potentials for ${ }^{1} S_{0}, S=-2, I=0$ sector as characteristic examples. To obtain $V_{i j}(r)$, we have used a simple analytic function with five parameters fitted to data of $V^{(\alpha)}$. The left panel of Fig. 4 shows the diagonal part of the potentials. We see that $\Lambda \Lambda$ and $N \Xi(I=0)$ channels are attractive while $\Sigma \Sigma(I=0)$ channel is repulsive. The attraction in the $\mathbf{1}$ channel is most reflected in the $N \Xi(I=0)$ channel, while the strong repulsion in the $\mathbf{8}_{s}$ channel is most reflected in the $\Sigma \Sigma(I=0)$, owing to its largest CG coefficient among the three channels. Nevertheless, all three diagonal potentials have a repulsive core originating from the $\boldsymbol{8}_{s}$ component. The right panel of Fig. 4 shows the off-diagonal parts of the potential matrix, which are comparable in magnitude to the diagonal ones. Since the off-diagonal parts are not negligible in this sector, full coupled channel analysis is necessary to study the observables.

Similarly, we have obtained potentials for all other sectors with different strangeness, iso-spin and spin, and find their features[7]. Since those features may remain in the flavor-SU(3)-broken physical world essentially, we expect our result provide useful hints on the behavior of hyperons $(\Lambda, \Sigma$ and $\Xi)$ in hypernuclei and in neutron stars [1].

\section{Summary and outlook}

We have performed 3-flavor full QCD simulations to study the general features of the $B B$ interaction in the flavor $S U(3)$ limit. From the NBS wave function measured on the lattice, we have extracted all six independent potentials in the $S$-wave at the leading order of the derivative expansion. We have found strong flavor-spin dependence of the $B B$ interactions. It turns out there exist variety of $B B$ interaction in $\mathrm{u}, \mathrm{d}$, s-quark three-flavor world, in contrast to $\mathrm{u}$, d-quark twoflavor world. In particular, $V^{\left(\mathbf{8}_{s}\right)}$ has a very strong repulsive core at a short distance, while $V^{(\mathbf{1})}$ is attractive at all distances. These features are consistent with the Pauli blocking effect among quarks previously studied in phenomenological quark models. This indicate that the quark Pauli blocking is essential for the repulsion in $B B$ interactions at a short distance. 
In the flavor singlet $B B$ channel, a bound state so called $H$-dibaryon is expected in phenomenological quark models[11]. The present lattice QCD data are insufficient to derive a definite conclusion on the $H$-dibaryon, because of a single and small lattice volume. Now we are performing lattice QCD simulations at flavor-SU(3)-point in larger volume. Nevertheless, using the potential $V^{(\mathbf{1})}(r)$ and solving the Schrödinger equation, a shallow bound state is found, suggesting a possibility of a bound $H$-dibaryon with baryon components $\Lambda \Lambda: \Sigma \Sigma: \Xi N=-\sqrt{1}: \sqrt{3}: \sqrt{4}$, in the $S U$ (3) limit world with heavy quark. In the real world, flavor $S U(3)$ breaking is considerable and the thresholds of the $S=-2, I=0$ sector are located in order $\Lambda \Lambda<\Xi N<\Sigma \Sigma$. In our test study of solving the Schrödinger equation with small baryon mass differences from a $2+1$ flavor lattice QCD simulation, a resonance state is found at an energy between $\Lambda \Lambda$ and $\Xi N$ threshold. If such a resonance exists in nature, it may explain the enhancement just above the $\Lambda \Lambda$ threshold recently reported in the KEK experiment [12]. However, further investigations in both theory and experiment are necessary to derive a definite conclusion.

\section{Acknowledgements}

The author thank the CPS for their lattice QCD simulation code [13], the CP-PACS and JLQCD Collaborations for their gauge configurations [10], which are provided through the JLDG / ILDG [9]. This research is supported in part by Grants-in-Aid for Scientific Research on Innovative Areas (No. 2004:20105001, 20105003) and the Large Scale Simulation Program No. 09-23 (FY2009) of the High Energy Accelerator Research Organization (KEK).

\section{References}

[1] Reviewed in O. Hashimoto and H. Tamura, Prog. Part. Nucl. Phys. 57 (2006), 564. Reviewed in J. Schaffner-Bielich, Nucl. Phys. A 835 (2010), 279, arXiv:1002.1658.

[2] R. Machleidt and I. Slaus, J. of Phys. G 27 (2001), R69, nucl-th/0101056.

[3] Reviewed in M. Oka, K. Shimizu and K. Yazaki, Prog. Theor. Phys. Suppl. No. 137 (2000), 1. Reviewed in Y. Fujiwara, Y. Suzuki and C. Nakamoto, Prog. Part. Nucl. Phys. 58 (2007), 439,

[4] Reviewed in T. A. Rijken, M. M. Nagels and Y. Yamamoto, Nucl. Phys. A 835 (2010), 160.

[5] N. Ishii, S. Aoki and T. Hatsuda, Phys. Rev. Lett. 99 (2007), 022001, nucl-th/0611096. S. Aoki, T. Hatsuda and N. Ishii, Prog. Theor. Phys. 123 (2010), 89, arXiv:0909.5585.

[6] H. Nemura [HAL QCD Collaboration and PACS-CS Collaboration], PoS LAT2009 (2009), 152

[7] T. Inoue et al. [HAL QCD Collaboration], Prog. Theo. Phys. Vol. 124 (2010), 591, arXiv:1007.3559

[8] K. Murano, N. Ishii, S. Aoki and T. Hatsuda, PoS LAT2009 (2009), 126, arXiv:1003.0530.

[9] Japan Lattice Data Grid (JLDG), http://www.jldg.org International Lattice Data Grid (ILDG), http://www.lqcd.org/ildg

[10] CP-PACS and JLQCD Collaboration, http://www.jldg.org/ildg-data/CPPACS+JLQCDconfig.html T. Ishikawa et al. [JLQCD Collaboration], Phys. Rev. D 78 (2008), 011502, arXiv:0704.1937.

[11] R. L. Jaffe, Phys. Rev. Lett. 38 (1977), 195, [Errata, 38 (1977), 617].

[12] C. J. Yoon et al., Phys. Rev. C 75 (2007), 022201.

[13] Columbia Physics System (CPS), http://qcdoc.phys.columbia.edu/cps.html 